\title{
An adaptive UKF filtering algorithm for self alignment in the swaying base of SINS
}

\author{
Su Wan-xin \\ Changchun Institute of Optics, Fine Mechanics and Physics, Chinese Academy of \\ Sciences,Changchun, 130033, China \\ ccswx@163.com
}

Keywords: UKF; SINS; Initial Alignment;Kalman; Adaptive Filter

\begin{abstract}
To the nonlinear model errors caused by the swaying base, unscented Kalman filters and Adaptive Unscented Kalman Filter (AUKF) are designed (UKF) for fine alignment respectively. In this paper, the adaptive estimation principle is introduced into the UKF algorithm. AUKF algorithm can balance automatically the right of the state information and observation information in the filtering result, so that to real-time adjust the covariance of the state vector and observation vector. The experimental results show: Compared with normal UKF algorithm, the adaptive UKF algorithm can eliminate the appearance of abnormal error and improve the accuracy and reliability of self alignment in the swaying base of SINS. In the following three direction east, north and day, the angle accuracy of misalignment is increased by 0.2 ', 0.2 ' and 5.0 '; convergence time is shortened by $10 \mathrm{~s}$, $28 \mathrm{~s}$ and $27 \mathrm{~s}$ respectively.
\end{abstract}

\section{Introduction}

Before the SINS work, the calculated navigation coordinate system needs to accurately align and track the real geographic coordinate system. When the carrier is in a static and non-interference environment on the indoor table, the high alignment accuracy can be obtained by the ordinary analytical method. In practical application, the random noise of the inertial device will increase when the carrier is disturbed by the wind and the engine vibration. Because of the disturbance of the external disturbance to the base, the output signal of the inertial sensing device contains the information of the earth's rotation angle velocity, and the carrier's shaking, so as to cause the dynamic error.

The self alignment in the swaying base of SINS is one of the most important research directions. The coarse alignment algorithms of anti interference are studied by literature [1], [2] and [3]. Literature [4] proposed another anti jamming self alignment algorithm for the rocking base of SINS. The algorithm can realize the self alignment without the coarse alignment at the same time in the interferences of angular motion and linear vibration. Kalman filtering method and $\mathrm{H} \infty$ filtering method were studied by literature [5] for the self alignment in the swaying base of SINS. Literature [6] is an application of Sage-Husa adaptive filtering algorithm to initial alignment of SINS static base. The above algorithms are mainly about the research of the coarse alignment of the swaying base and the uncertain statistical properties of the system noise and observation noise.

A nonlinear error model is also caused by the shaking of the base. At this time, we need to carry out nonlinear filtering. The methods of nonlinear self alignment were determined by literature [7]. By comparing the KF, EKF and UKF filtering algorithm, in the case of larger azimuth misalignment angle,the KF is no longer applicable, and the effect of UKF is better than EKF. Application process of UKF algorithm in SINS initial alignment was studied by literature [8].

UKF filter is a good nonlinear filter, but it is sensitive to the choice of the initial value. When the abnormal disturbance, it will affect the filtering results. In view of this situation,in this paper, the unscented Kalman filter (UKF) and the improved adaptive UKF (AUKF) are designed and compared for the self alignment of the swaying base SINS. Compared with normal UKF algorithm, the adaptive 
UKF algorithm can eliminate the appearance of abnormal error and improve the accuracy and reliability of self alignment in the swaying base of SINS.

\section{Establishing State and Observation Equation}

\section{Coarse alignment process}

Self alignment is divided into coarse alignment and fine alignment. The task of coarse alignment is to quickly obtain a rough initial attitude matrix. Coarse alignment is usually referred to as analytical alignment. In coarse alignment stage, depending on the observation value of the acceleration of gravity g and the Earth's rotation angular velocity $\omega_{i e}$, the attitude matrix from the carrier coordinate system to the navigation system is directly estimated.

In the initial alignment of the static base, we chose the carrier's navigation coordinate system (n) for $(E, N, U)$ geographic coordinate system $(t)$.The component of the acceleration $g$ vector was meatured by accelerometer in the carrier coordinate system (b). Similarly, the component of the the Earth's rotation angular velocity $\omega_{i e}$ was meatured by gyroscope.

In the case of local latitude, the component of the gravity vector and the earth self rotation angular velocity vector are known in the geographic coordinate system as below:

$$
\begin{aligned}
& g^{n}=\left[\begin{array}{lll}
0 & 0 & -g
\end{array}\right]^{T} \\
& \omega_{i e}^{n}=\left[\begin{array}{lll}
0 & \omega_{i e} \cos L & \omega_{i e} \sin L
\end{array}\right]^{T}
\end{aligned}
$$

The conversion relationship between carrier coordinate system and navigation coordinate system is as in Eq. (1):

$$
\begin{aligned}
& g^{b}=C_{n}^{b} g^{n} \\
& \omega_{i e}^{b}=C_{n}^{b} \omega_{i e}^{n}
\end{aligned}
$$

Because $C_{n}^{b}$ is orthogonal, it is satisfied with $C_{b}^{n}=\left(C_{n}^{b}\right)^{-1}=\left(C_{n}^{b}\right)^{T}$.

By vector multiplication, we got the other 3 vectors: $g \times \omega_{i e}, g \times \omega_{i e} \times g, \omega_{i e} \times g \times \omega_{i e}$ with two methods to discuss. After the analysis of coarse alignment, the obtained attitude matrix was corrected by the misalignment angle and became more accurately. The correction procedure is as below:

$$
\dot{C}_{b}^{n}=(I-\phi) C_{b}^{n}
$$

Here, I is the unit matrix. $\phi$ is the correction matrix of the misalignment angle.

First method is shown below:

$$
\phi=\left[\begin{array}{ccc}
0 & -\phi_{U} & \phi_{E} \\
\phi_{U} & 0 & -\phi_{N} \\
-\phi_{E} & \phi_{N} & 0
\end{array}\right]
$$




$$
\begin{aligned}
& V_{1}=\left[\begin{array}{lll}
g & \omega_{i e} & g \times \omega_{i e}
\end{array}\right] \\
& C_{b}^{n}=\left[\begin{array}{lll}
C_{11} & C_{12} & C_{13} \\
C_{21} & C_{22} & C_{23} \\
C_{31} & C_{32} & C_{33}
\end{array}\right]=\left[\begin{array}{c}
\left(g^{n}\right)^{T} \\
\left(\omega_{i e}^{n}\right)^{T} \\
\left(g^{n} \times \omega_{i e}^{n}\right)^{T}
\end{array}\right]^{-1}\left[\begin{array}{c}
\left(g^{b}\right)^{T} \\
\left(\omega_{i e}^{b}\right)^{T} \\
\left(g^{b} \times \omega_{i e}^{b}\right)^{T}
\end{array}\right] \\
& \delta a^{n}=C_{b}^{n} \delta a^{b}=\left[\begin{array}{lll}
\delta a_{E} & \delta a_{N} & \delta a_{U}
\end{array}\right]^{T} \\
& \delta \omega^{n}=C_{b}^{n} \delta \omega^{b}=\left[\begin{array}{lll}
\delta \omega_{E} & \delta \omega_{N} & \delta \omega_{U}
\end{array}\right]^{T} \\
& \phi_{E}=\frac{1}{2}\left[\frac{\delta a_{N}}{g}+\frac{\delta a_{U}}{g} \tan L+\frac{\delta \omega_{U}}{\omega_{i e} \cos L}\right] \\
& \phi_{N}=-\frac{\delta a_{E}}{g}, \phi_{U}=-\frac{\delta a_{E}}{g} \tan L-\frac{\delta \omega_{E}}{\omega_{i e} \cos L}
\end{aligned}
$$

Second Method is as follows:

By comparing both methods, the results show that the east misalignment angle in the second is not affected by the output error of the up gyro and accelerometer. In strapdown inertial sensors, the precision of the accelerometer is much higher than that of the gyroscope. Besides, the output error of the up gyro is an important factor to influence the east misalignment angle. Based on the above reasons, the second method is better than the first.

$$
\begin{aligned}
& V_{2}=\left[\begin{array}{lll}
g & g \times \omega_{i e} & \left(g \times \omega_{i e}\right) \times g
\end{array}\right] \\
& C_{b}^{n}=\left[\begin{array}{lll}
C_{11} & C_{12} & C_{13} \\
C_{21} & C_{22} & C_{23} \\
C_{31} & C_{32} & C_{33}
\end{array}\right]=\left[\begin{array}{c}
\left(g^{n}\right)^{T} \\
\left(g^{n} \times \omega_{i e}^{n}\right)^{T} \\
\left(\left(g^{n} \times \omega_{i e}^{n}\right) \times g^{n}\right)^{T}
\end{array}\right]^{-1}\left[\begin{array}{c}
\left(g^{b}\right)^{T} \\
\left(g^{b} \times \omega_{i e}^{b}\right)^{T} \\
\left(\left(g^{b} \times \omega_{i e}^{b}\right) \times g^{b}\right)^{T}
\end{array}\right] \\
& \delta a^{n}=C_{b}^{n} \delta a^{b}=\left[\begin{array}{lll}
\delta a_{E} & \delta a_{N} & \delta a_{U}
\end{array}\right]^{T} \\
& \delta \omega^{n}=C_{b}^{n} \delta \omega^{b}=\left[\begin{array}{lll}
\delta \omega_{E} & \delta \omega_{N} & \delta \omega_{U}
\end{array}\right]^{T} \\
& \phi_{E}=\frac{\delta a_{N}}{g}, \phi_{N}=-\frac{\delta a_{E}}{g}, \phi_{U}=-\frac{\delta a_{E}}{g} \tan L-\frac{\delta \omega_{E}}{\omega_{i e} \cos L}
\end{aligned}
$$

\section{Establishing State and Observation Equation}

After coarse alignment, the initial attitude matrix is established. The goal of the fine alignment is to update the attitude matrix $C_{b}^{n}$ to make sure that the navigation system is gradually close to a real geographic coordinate system.

For nonlinear filtering algorithm, the state and observation equations are firstly established. The state variables and variables of the system are as follows:

$$
\begin{aligned}
\mathbf{X} & =\left[\delta \dot{V}_{E}, \delta \dot{V}_{N}, \dot{\varphi}_{E}, \dot{\varphi}_{N}, \dot{\varphi}_{U}, \nabla_{x}, \nabla_{y}, \varepsilon_{x}, \varepsilon_{y}, \varepsilon_{z}\right]^{T} \\
Z & =\left[\delta \dot{V}_{E}, \delta \dot{V}_{N}\right]^{T}
\end{aligned}
$$

The observation value is the horizontal velocity error $\delta \dot{V}_{E}, \delta \dot{V}_{N}$ in the navigation coordinate system; $\dot{\varphi}_{E}, \dot{\varphi}_{N}, \dot{\varphi}_{U}$ are misalignment angles. $\nabla_{x}, \nabla_{y}$ are random constant bias of horizontal accelerometer in the carrier coordinate system; $\varepsilon_{x}, \varepsilon_{y}, \varepsilon_{z}$ are random constant drift of gyroscope in three directions of the system.

The nonlinear state and the observation equation of the fine alignment of the SINS system is in Eq.(3):

$$
\begin{aligned}
& \dot{X}=A X+f(x)+G W \\
& Z=H X+V=\left[\begin{array}{ll}
I_{2 \times 2} & 0_{2 \times 8}
\end{array}\right] X+V
\end{aligned}
$$

The state equation is divided into linear part $A X$ and nonlinear part $\mathrm{f}(\mathrm{x})$. 
Among them:

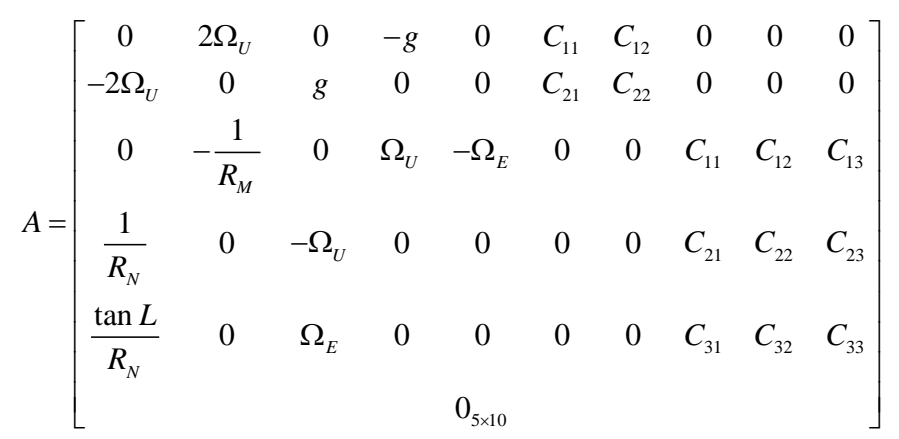

$$
\begin{aligned}
& f(x)=\left[\begin{array}{l}
\quad 0_{2 \times 1} \\
-\Omega_{N} \sin \phi_{U} \\
\Omega_{N}\left(1-\cos \phi_{U}\right) \\
\Omega_{N}\left(-\phi_{N} \sin \phi_{U}+\phi_{E} \cos \phi_{U}\right) \\
0_{5 \times 1}
\end{array}\right], \quad G=\left[\begin{array}{ccccc}
C_{11} & C_{12} & 0 & 0 & 0 \\
C_{21} & C_{22} & 0 & 0 & 0 \\
0 & 0 & C_{11} & C_{12} & C_{13} \\
0 & 0 & C_{21} & C_{22} & C_{23} \\
0 & 0 & C_{31} & C_{32} & C_{33} \\
& & 0_{5 \times 5} &
\end{array}\right]
\end{aligned}
$$

$\Omega_{U}=\omega_{\mathrm{ie}} \sin L, \Omega_{N}=\omega_{\mathrm{ie}} \cos L . \mathbf{W}=\left[\begin{array}{llllll}w_{\delta V_{E}^{n}} & w_{\delta V_{N}^{n}} & w_{\phi x} & w_{\phi y} & w_{\phi z} & 0_{1 \times 5}\end{array}\right]^{T}$ is zero mean Gaussian white noise of the system. $V=\left[\begin{array}{ll}v_{\delta V_{E}} & v_{\delta V_{N}}\end{array}\right]^{T}$ are the zero mean measurement of Gaussian white noise. $\mathrm{L}$ is the local latitude.

\section{Establishing Error Model}

Considering the fine alignment time is short, gyroscopes and accelerometers error model were both approximately considered as constant drift with white noise: $\nabla=\nabla_{b}+w_{a}, \quad \varepsilon=\varepsilon_{b}+w_{g}$.

$\nabla_{b}=0, w_{a}$ is Gaussian white noise of accelerometers; $\varepsilon_{b}=0, w_{g}$ is gaussian white noise of gyroscopes.

In general, for the horizontal misalignment angle $\phi_{E}$ and $\phi_{N}$ are small after coarse alignment, $\sin \phi_{E}$ and $\sin \phi_{N}$ are approximately equal to $\phi_{E}$ and $\phi_{N} \cdot \cos \phi_{E}$ and $\cos \phi_{N}$ are approximately close to 1 . However, the azimut misalignment angle is too large to be estimated. The initial alignment of the static base on the earth surface is: $f_{E}=f_{N} \approx 0, f_{U}=-g$.

The error equation of IMU inertial navigation system is as below:

$$
\left\{\begin{array}{l}
\delta \dot{V}_{E}=-g\left(\varphi_{N} \cos \varphi_{U}+\varphi_{E} \sin \varphi_{U}\right)+2 \omega_{i e}(\sin L) \delta V_{N}+\nabla_{E} \\
\delta \dot{V}_{N}=-g\left(\varphi_{N} \sin \varphi_{U}-\varphi_{E} \cos \varphi_{U}\right)-2 \omega_{i e}(\sin L) \delta V_{E}+\nabla_{N} \\
\dot{\varphi}_{E}=-\sin \varphi_{U} \omega_{i e} \cos L+\varphi_{N} \omega_{i e} \sin L-\delta V_{N} / R_{m}+\varepsilon_{E} \\
\dot{\varphi}_{N}=\left(1-\cos \varphi_{U}\right) \omega_{i e} \cos L-\varphi_{E} \omega_{i e} \sin L+\delta V_{E} / R_{n}+\varepsilon_{N} \\
\dot{\varphi}_{U}=\left(-\varphi_{N} \sin \varphi_{U}+\varphi_{E} \cos \varphi_{U}\right) \omega_{i e} \cos L+\delta V_{E} \tan L / R_{n}+\varepsilon_{U}
\end{array}\right.
$$

$\omega_{i e}$ is the angular velocity of the Earth's rotation; $\varepsilon_{E}, \varepsilon_{N}, \varepsilon_{U}$ show gyro error of the navigation coordinate system; $\mathrm{L}$ is the geographic latitude; $\mathrm{h}$ is the geographic height; $R_{m}$ is the radius of curvature of meridian; $R_{n}$ is the prime vertical radius of curvature. $\nabla_{E}$ and $\nabla_{N}$ are the horizontal error of the accelerometer in the navigation coordinate system.

\section{UKF Algorithm and AUKF Algorithm}

\section{Establishing Error Model}

In order to improve the effect of nonlinear filtering, Julier proposed an unscented Kalman filter (Unscented Kalman Filter, UKF) method to estimate the nonlinear filtering problem. First, this 
method processes Unscented Transform (U tranformation) on the state equation, estimate the filtering result after the UT, in turn reduce the estimation error. Unlike the Extended Kalman Filter (EKF) to linearize the nonlinear system model or calculte Jacobian matrix, UKF deal directly with the nonlinear model, which becomes increasingly widely-used in navigation system.

For one step estimate equation, UKF use the assured sample strategy directly to handle nonlinear deliver of the system's mean value and covariance error. The design step of UKF is described as below.

Initializing:

$$
\left.\begin{array}{rl}
\left(\hat{x}_{0}\right. & =E\left[x_{0}\right] \\
P_{0} & =E\left[\left(x_{0}-\hat{x}_{0}\right)\left(x_{0}-\hat{x}_{0}\right)^{T}\right]
\end{array}\right\}
$$

Calculating Sigma Point and Corresponding Weight:

$$
\left.\begin{array}{l}
\chi_{0, k-1}=\hat{x}_{k-1} \\
\chi_{i, k-1}=\hat{x}_{k-1}+\sqrt{(n+\lambda) P_{k-1}},(i=1,2, \cdots, n) \\
\chi_{i, k-1}=\hat{x}_{k-1}-\sqrt{(n+\lambda) P_{k-1}},(i=n+1, n+2, \cdots, 2 n) \\
w_{0}^{(m)}=\lambda /(n+\lambda) \\
w_{0}^{(c)}=\lambda /(n+\lambda)+\left(1-\xi^{2}+\eta\right) \\
w_{i}^{(m)}=w_{i}^{(c)}=1 /(2(n+\lambda)),(i=1,2, \ldots, 2 n)
\end{array}\right\}
$$

$\lambda=\xi^{2}(\mathrm{n}+\kappa)-n$ is a scaling factor; $\xi$ can determine the value range of sampling points, usually chosen between a small positive number between $0-1$, such as $1 \mathrm{e}-3 ; \kappa$ is a constant, generally 0 or 3- $\mathrm{n}$; $\eta$ depends on distribution of state variables. $\eta=2$ is optimal when ${ }^{X}$ is subject to Gauss distribution. $w_{i}^{(m)}$ and $w_{i}^{(c)} \mathrm{i}$ are the weights of the mean value and variance.

Updating time:

$$
\left.\begin{array}{l}
\chi_{i, k \mid k-1}=f\left(\chi_{i, k-1}\right) \\
\hat{x}_{k}^{-}=\sum_{i=0}^{2 n} w_{i}^{(m)} \chi_{i, k \mid k-1} \\
P_{k}^{-}=\sum_{i=0}^{2 n} w_{i}^{(c)}\left[\chi_{i, k \mid k-1}-\hat{x}_{k}^{-}\right]\left[\chi_{i, k \mid k-1}-\hat{x}_{k}^{-}\right]^{T}+Q_{k} \\
Z_{i, k \mid k-1}=H \times \chi_{i, k \mid k-1} \\
\hat{Z}_{k}^{-}=\sum_{i=0}^{2 n} w_{i}^{(m)} Z_{i, k \mid k-1}
\end{array}\right\}
$$

$\mathrm{Q}_{\mathrm{k}}$ is the variance of the system noise $\mathrm{w}$ at $\mathrm{k}$ moment.

Updating Measurement:

$$
\begin{aligned}
& P_{Z_{k}, Z_{k}}=\sum_{i=0}^{2 n} w_{i}^{(c)}\left[Z_{i, k \mid k-1}-\hat{Z}_{k}^{-}\right]\left[Z_{i, k \mid k-1}-\hat{Z}_{k}^{-}\right]^{T}+R_{k} \\
& P_{x_{k}, Z_{k}}=\sum_{i=0}^{2 n} w_{i}^{(c)}\left[X_{i, k \mid k-1}-\hat{x}_{k}^{-}\right]\left[Z_{i, k \mid k-1}-\hat{Z}_{k}^{-}\right]^{T} \\
& K_{k}=P_{x_{k}, Z_{k}} \times P_{Z_{k}, Z_{k}}^{-1} \\
& \hat{x}_{k}=\hat{x}_{k}^{-}+K_{k}\left(Z_{k}-\hat{Z}_{k}^{-}\right) \\
& P_{k}=P_{k}^{-}-K_{k} \times P_{Z_{k}, Z_{k}} \times K_{k}^{T}
\end{aligned}
$$

$\mathrm{Z}_{\mathrm{k}}$ is the measurement vector at $\mathrm{k}$ moment; $\mathrm{R}_{\mathrm{k}}$ is the variance of the measurement at $\mathrm{k}$ moment. 
By analyzing the calculation steps of UKF, it's assumed that the method has the following advantages.

The calculation accuracy of nonlinear Gaussian distribution statistics achieves at least third order compared with second order for non-Gaussian distribution;

There is no need to make a clear understanding of the specific form of the nonlinear function, derivatives and Jacobians matrix;

The whole complexity of the algorithm equals to EKF, but UKF can lessen the influence of truncation error of the higher-order terms and is easy to implement.

UKF filtering method to estimate the nonlinear filtering problem not only provides the above unique advantages, but also overcome a series of problems existing in EKF. In practice, however, it's discovered that UKF is more sensitive to the choice of the initial value. An error in the initial value will directly affect the outcome of filtering result. Moreover, even if the initial value is reasonable, the existence of various disturbance error and uncertainty of statistical noise models will also affect the accuracy of UKF filtering. This paper, based on the principle of adaptive algorithm, put forward a brand new filter estimation method to achieve fine alignment.

\section{Adaptive UKF Algorithm}

Adaptive UKF algorithm and traditional UKF have basically the same structure. Some adjustments are performed as in Eq.(9):

$$
\left.\begin{array}{l}
P_{Z_{k}, Z_{k}}=\frac{1}{a_{k}} \sum_{i=0}^{2 n} w_{i}^{c}\left[Z_{i, k \mid k-1}-\hat{Z}_{k}^{-}\right]\left[Z_{i, k \mid k-1}-\hat{Z}_{k}^{-}\right]^{T}+R_{k} \\
P_{x_{k}, Z_{k}}=\frac{1}{a_{k}} \sum_{i=0}^{2 n} w_{i}^{c}\left[\chi_{i, k \mid k-1}-\hat{X}_{k}^{-}\right]\left[Z_{i, k \mid k-1}-\hat{Z}_{k}^{-}\right]^{T} \\
P_{k}=\frac{1}{a_{k}} P_{k}^{-}-K_{k} \times P_{Z_{k}, Z_{k}} \times K_{k}^{T}
\end{array}\right\}
$$

$a_{k}$ is the adaptive factor, the initial value is taken as 1 , value range $0 \leq a_{k} \leq 1$. If the value of $a_{k}$ is reasonable, it is possible to balance the ratio between the prediction and measurement information in the system model. $a_{k}$ is constructed as in Eq.(10):

$$
a_{k}=\left\{\begin{array}{cl}
1 & \operatorname{tr}\left(V_{k} V_{k}^{T}\right) \leq \operatorname{tr}(P) \\
\frac{\operatorname{tr}(P)}{\operatorname{tr}\left(V_{k} V_{k}^{T}\right)} & \operatorname{tr}\left(V_{k} V_{k}^{T}\right)>\operatorname{tr}(P)
\end{array}\right.
$$

Taking a prediction residual $V_{k}=Z_{k}-\hat{Z}_{k}^{-}$, covariance matrix is:

$$
P=\sum_{i=0}^{2 n} w_{i}^{(c)}\left[Z_{i, k \mid k-1}-\hat{Z}_{k}^{-}\right]\left[Z_{i, k \mid k-1}-\hat{Z}_{k}^{-}\right]^{T}
$$

The above impoved UKF algorithm shows that when there exists bias in initial value selection or abnormal disturbance in system model, $a_{k}$ will be less than 1 , which means the weight of forecast information in system model is as low as possible in the final filtered result. When the prediction of the system model is obviously abnormal, $a_{k}$ will approach 0 . That is to say the system model forecast information should be abandoned. Obviously, $a_{k}$ is able to adaptively adjust $\hat{x}_{k}^{-}$by the predicted residual $V_{k}$ and measurement information $\hat{Z}_{k}^{-}$.

\section{Experimental Analysis}

Initial parameters were set as below: Latitude $\mathrm{L}=43.50^{\circ}$; the initial value of the state variable $\mathrm{x}$ is 0 ; the initial misalignment angles after coarse alignment are $\left[\begin{array}{lll}0.324^{\circ} & 0.358^{\circ} & 3.31^{\circ}\end{array}\right]$; gyro constant drift $1^{\circ} / \mathrm{h}$, random drift $0.5^{\circ} / \mathrm{h}$; the initial bias of accelerometer in three directions are $0.5 \mathrm{mg}$, random deviations is $0.1 \mathrm{mg}$; horizontal velocity error is $0.1 \mathrm{~m} / \mathrm{s}$. Experimental results are shown in Figure 1, Figure 2, Figure3. 


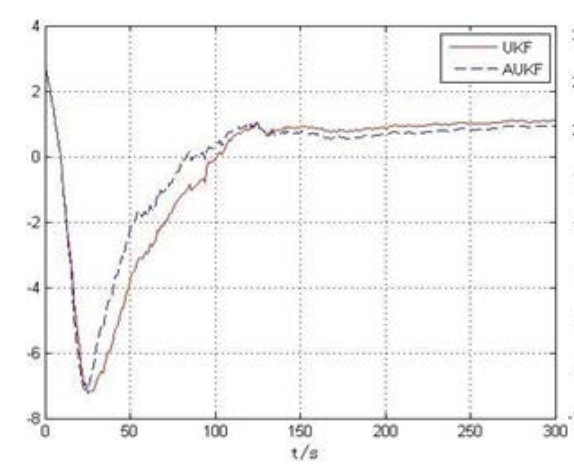

Figure $1 \Phi_{\mathrm{E}}$ angle

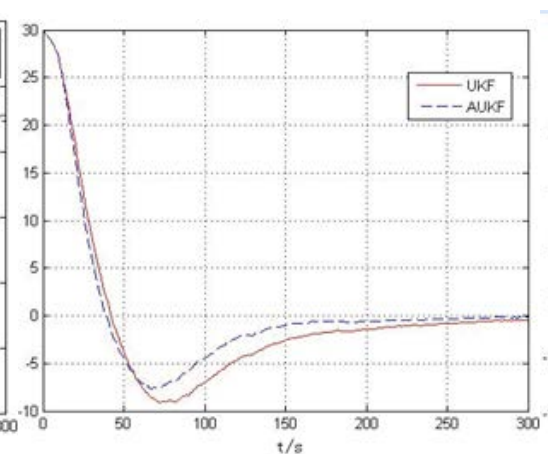

Figure $2 \Phi_{\mathrm{N}}$ angle

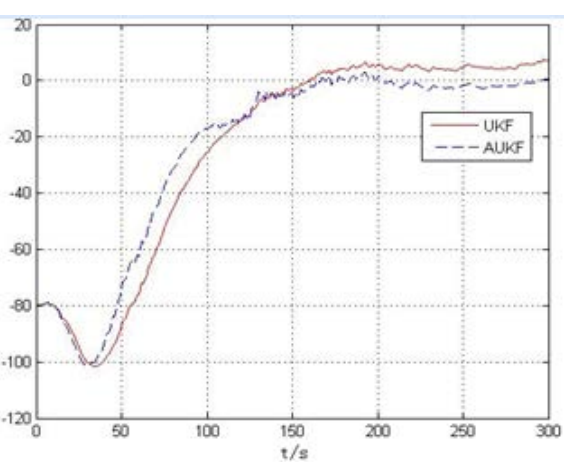

Figure3 $\Phi_{\mathrm{U}}$ angle

The figures show that the convergence precision of three misalignment angles are 1.2', $0.3^{\prime}$ and 6.0' when using conventional UKF filtering algorithm. The convergence precision of adaptive UKF (AUKF) are improved by 1.0', 0.1' and 1.0' . In alignment, the convergence time of the AUKF's three misalignment angles were 110s, 152s and 173s compared to 120s, 180s and 200s of UKF.

\section{Conclusion}

For the self alignment of the swaying base SINS, the accuracy and convergence rate of the misalignment angles were improved when using the AUKF algorithm compared with UKF in all three directions: East, North and Up. The accuracy was increased by $0.2^{\prime}, 0.2^{\prime}$ and $5.0^{\prime}$; convergence time shortened by 10s, 28s and 27s respectively. The reason is that when the AUKF filter is disturbed by the initial value error and the system model, the covariance of the state vector and the observation vector can be adjusted in real time by the weight ratio of the state and observation information in the filtering results.

\section{References}

[1] Zhao C S, Qin Y Y, Wei L. A gravity-based anti-interference coarse alignment algorithm[J]. J of Astronautics, 2010, 31(10): 2335-2339..

[2] Sun F, Sun W. Research on coarse alignment ofrotary SINS on a swing base[J]. Chinese J of Scientific Instrument, 2010, 31(4): 929-936.

[3] YUAN Tao, QU Zhigang.Algorithm Research on Independent Alignment of Aircraft Carrier s SINS. Journal of Projectiles, Rockets, Missiles and Guidance, 2013,Vol.33:24-30.

[4] WANG Yue-gang, YANG Jia-sheng. SINS anti-interference self-alignment algorithm for the swaying base. Control and Decision, 2014, Vol. 29:546-550.

[5] HUANG Chun- mei,SUN Xiao-hui. Two algorithms for auto alignment in rocki ng base of SI NS, Journal of Chang c hun University of Technology(Natural Science Edition, 2013,Vol.34:711-714.

[6] SU Wan-xin.Application of Sage-Husa adaptive filtering algorithm for high precision SINS stationaryin initial alignment. Journal of Chinese Inertial Technology, 2010,Vol.18:12-16.

[7] Shen Liyun. KEY TECHNOLOGIES RESEARCH ON INDEPENDENT ALIGNMENT OF STRAP-DOWN INERTIAL NAVIGATION SYETEM, Harbin Institute of Technology, June, 2014.

[8] Zhou Benchuan ,Cheng Xianghong ,Robust UKF algorithm in SINS initial alignment[J]. Journal of Southeast University ( English Edition),2011,27(1):56-65. 